\title{
Awareness and perception of women of reproductive age (15-49) regarding breast cancer at Okuryangava clinic, Namibia
}

\author{
Nghitanwa Emma Maano*, Haitembu Teopolina, Hatupopi Saara Kerthu \\ Department of Midwifery Science, School of Nursing, Faulty of Health Sciences, University of Namibia, Namibia
}

Received: August 16, 2019

DOI: $10.5430 / \mathrm{cns} . v 8 \mathrm{n} 1 \mathrm{p} 9$
Accepted: October 28, 2019

Online Published: November 25, 2019

\begin{abstract}
Globally cancer of the breast is a challenge, and in Namibia it ranks the third common type of cancer. The purpose of this study was to assess the awareness and perception of women of reproductive age (15-49) regarding breast cancer at Okuryangava clinic. Ethical clearance for the study was obtained from the Ministry of Health and Social Services of Namibia and the University of Namibia research ethical committee. Qualitative and exploratory study design was conducted. After obtained the written consent from participants, data were collected among 10 women with interview guide through face to face interview. The sample size was determined by data saturation. Data were analyzed according to Tesch's eight steps of data analyses that led to the formation of themes and sub themes. The study found out that majority of women at Okuryangava clinic had awareness about breast cancer. Women had different perception about breast cancer, some believe that breast cancer is curable while others perceive it as a fatal disease. The study also found that participants have knowledge on breast cancer risk factors which may assist in preventive measuresand awareness. The study recommends that the Ministry of Health and Social Services of Namibia should ensure conducting an awareness campaigns to educate the community regarding breast cancer.
\end{abstract}

Key Words: Awareness, Perception, Breast cancer, Reproductive age

\section{INTRODUCTION}

Breast cancer is a disease that can affect people of different race, socio-economic status or culture. Globally, breast cancer is a health problem and is mostly affecting women aged 15-49 years old. ${ }^{[1]}$ The common types of cancer of the breast include ductal carcinoma, lobular carcinoma and invasive breast cancer. ${ }^{[2]}$ According to Lee and Han, ${ }^{[3]}$ breast cancer occurs due to the uncontrollability of multiplication of cells in the body. There is a challenge in breast cancer treatment in developing countries as most of the cancers are detected at later stage due to poor knowledge of breast examination among women which leads to the delay in early detection as well as lack of health facilities responsible for cancer screening. ${ }^{[4]}$ However, early diagnose of breast cancer can effectively improve the chance in early detection that may positively influence the treatment outcome, quality of life improvement and prevent fatalities. ${ }^{[5]}$

The World Health Organization (WHO), reported that about 14.1 million new cases of cancer is diagnosed annually and that more than half of these occur in developing countries. According to Ferlay, Mathers \& Rebelo, ${ }^{[6]}$ breast cancer is the second most common cancer in women worldwide, with

\footnotetext{
*Correspondence: Nghitanwa Emma Maano; Email: enghitanwa@unam.na; Address: Department of Midwifery Science, School of Nursing, Faulty of Health Sciences, University of Namibia, Namibia.
} 
nearly 1.7 million new cases diagnosed in 2012 and the fifth most common cause of death in women. In Kenya, Muthoni and Miller ${ }^{[7]}$ conducted a study among women who are not diagnosed of cancer, which found out that most women were unable to show their knowledge on the contributing factors as well as clinical manifestations of breast cancer. Furthermore, the study showed that women were able to state some risk that may make them susceptibility to breast cancer. These authors further stated that, although participants have not received health advice regarding breast cancer, being seen a acquaintances with breast cancer make them aware of breast cancer. ${ }^{[7]}$ Poor awareness as well as information about breast cancer is a challenge that causes women to seek medical attention when the disease is already at its advanced stages and this will lead to poor prognosis of the disease. ${ }^{[8]}$

The Cancer Association of Namibia stated that women delay seeking assistance when experience breast cancer manifestations. ${ }^{[9]}$ This implies lack of perception on the importance of prompt identification and early treatment of breast cancer. As a result unavailability of health facilities responsible for cancer screening, reproductive health changes and physical inactivity could be linked to the burden associated with cancer-related mortality. ${ }^{[10]}$ The Namibia National Cancer Registry, ${ }^{[9]}$ indicates a $29 \%$ increase in recorded cancer over the past five years. The most common type of cancer in Namibia is skin cancer. However, breast cancer remains the number one type of cancer in women, followed by cervical cancer. Poor awareness and information about breast cancer is the problem that causes women to seek medical attention when the disease is already at its advanced stages and this will lead to poor prognosis of the disease. ${ }^{[8]}$

\subsection{Purpose of the study}

The purpose of this study was to assess the awareness and perception of women of reproductive age (15-49 years) at Okuryangava clinic regarding breast cancer.

\subsection{Objectives of the study}

- To assess the awareness regarding breast cancer among women of reproductive age (15-49) at Okuryangava clinic

- To explore the perception regarding breast cancer among women of reproductive age (15-49) at Okuryangava clinic

- To explore the factors that contribute to breast cancer

\section{Methods}

Qualitative, exploratory study design was used to conduct this study. This design was appropriate for this study because it allows researchers to assess and explore the aware- ness, perception and factors regarding breast cancer from the participant's point of view.

The population of this study consisted of women aged 15-49 years, attending the family planning services at Okuryangava clinic in November 2018. The researchers employed a nonprobability sampling coupled with the use of the purposive sampling method to produce a sample from population. Purposive sampling was used selecting every second woman. The researchers continued to select respondents until the point of redundancy or saturation is reached. This point is obtained when new interviews no longer yield new information. Ten women were selected for in-depth interviews. Interview was conducted by the researchers in the office at Okuryangava clinic. The inclusion criteria include women aged 15-49 years visiting Okuryangava clinic seeking family planning who agreed to participate. In addition, all women who did not give consent to participate in the study were excluded.

Data were collected among 10 participants through face to face in depth interviews using interview guide. The interview guide consists of the following main questions: participant's awareness on breast cancer; participant's perception regarding breast cancer and contributing factors to breast cancer. All interviews were audio recorded with the respondents' permissions. Field notes were also used by the researchers to record non verbal communication and to invite the respondents to give more information or explain the first response. At the end of the discussion, member checking was conducted to confirm with the respondents whether what was recorded was a true reflection of what they said or check whether the researchers understood them correctly. Data saturation was reached at ten respondents as there were no new ideas being generated. Data were recorded with a voice recorder, translated, transcribed coded in line with Tesch' s method of data analysis. A list of all similar codes were grouped together to form themes and then sub-themes. Trustworthiness was ensured through the use of the Lincoln \& Guba's (1985) criteria: credibility, transferability, dependability and confirmability. Credibility was ensured by lengthy exposure with respondents and debriefing of members made soon after data collection, which ensured that the respondents could see transcripts of the interviews to confirm whether they were exact reflection of what they had said during the interview. Transferability was ensured by collecting data up to saturation point. In addition, the researchers clearly indicated the detailed descriptions of the results. Dependability in this study was enriched by providing a dense description of the research approaches used and its implementation, as well as by describing the steps that were taken to collect data and how the data were analysed. Confirmability was ensured 
by discussing the research process and findings with respondents, with the researchers upholding reflexivity by writing down their beliefs and perceptions to avoid influencing the findings and decisions of the study.

\subsection{Research ethics}

Authorization to conduct the study was obtained from the Ministry of Health and Social Services as well as from the University of Namibia research ethical committee. The researchers also got the permission from the registered nurse in charge of Okuryangava clinic. Informed consent form was signed by each participant before data collection process to confirm that they have agreed to participate in to the study. Privacy and confidentiality were ensured by allocating codes and numbers for participants and not to reveal their names. Participants were informed their right to withdraw from the study anytime if they wish. By avoiding harm to the participants, the questions were carefully structured and participants were monitored for any sign of distress during data collection. All participants had an equal chance of being selected to participate in the study.

\section{RESULTS}

\subsection{Demographic characteristics of the participants}

Participants interviewed were aged between 18 and 30 years. Most of the participants were single, only one participant was married. One participant failed grade 9 , four participants failed their grade 10, four of the participants are students at the university and only one participant completed school. Nine participants were unemployed and only one working.

Three themes and six sub-themes emerged from the study as shown in Table 1.

Table 1. Theme 1: Participants awareness of breast cancer

THEME
Theme 1: Participants awareness of breast cancer
Theme 2: Participants perceive breast cancer differently

Theme 3: Participants knowledge about the risk factors of breast cancer

\section{SUBTHEME}

Sub-theme 1: Mixed perception Sub-theme 2: Negative perception Sub-theme 1: Alcohol and smoking Sub-theme 2: Hormonal replacement therapy Sub-theme 3: Age and genetic factors Sub-theme 4: Sun exposure
Participants indicated that they were aware of breast cancer as a disease commonly affect women of reproductive age. Participants also stated the importance of conducting self breast examination in order to detect abnormal lumps on their breasts which sometimes turn to be cancerous. One participant stated as follow:

“' 'aura, mhm' I think breast cancer is a disease, it's a breast disease that affect women of child bearing age. You can tell that you have a disease by getting breast discharges or maybe you have lumps in your breast... that's all."

Another participant replies:

"When you are checking your breast if there is a lump or something that is not normal."

Regarding breast examination, one participants was quoted:

“Breast examination it's when you examine your breast to feel for lumps if you have any lumps, I think it's just the same as screening but then maybe screening has to do much with the hospital when you go to the hospital just to get breast screening, when the nurses are the one examining our breast to feel for lumps."

\section{Theme 2: Participants perceive breast cancer differently}

Participants perceive breast cancer differently, positive or negative, although there were some participants with mixed perception.

\section{Sub-themes: Mixed perception}

Participate were asked how they perceive breast cancer and here is their responses with both positive and negative feelings as follow:

"Breast cancer is the dangerous disease, especially when it's left untreated but the good part of it, it can be treated like it can be cured when detected earlier."

Another participant stated:

"Breast cancer is a very dangerous disease but if they treat it early it can be cured but if they didn't find it early you can die."

\section{Negative perception: Very dangerous, killing disease}

Most participants stated that breast cancer is very dangerous disease and can cause death. 
One participant stated that:

" 'aaaa' what I understand about breast cancer, on my own option is that it's a killing disease if not detected earier. 'aaa...' there is a treatment they do at the hospital just to prolong but doesn't cure exactly".

Another participant mentioned that:

"My perception about breast cancer is that breast cancer is a serious disease but it kills people but when the person is lucky it can be operated on and the person can live for years."

Another participant was quoted:

"But we have to understand that whether the person get treated for breast cancer or not, it is a serious disease."

\section{Theme 3: Participants are aware about the predisposing factors of breast cancer}

\section{Sub-themes: Alcohol and smoking}

Most participants indicated that alcohol is a predisposing factor for breast cancer and most women who smoke are more likely to drink alcohol. Smoking is indicated as major factor which contributed to breast cancer.

One participant reply:

"You can get it from a family when someone has it in your family or sometimes you can get it from drinking alcohol or smoking."

Another participant stated:

"' aaa' I think breast cancer can be caused by too much alcohol consumptions and smoking."

\section{Sub-themes: Hormonal replacement therapy}

One participant indicated that taking hormones to replace missing hormones for five years and more raises the risk for breast cancer.

Participant was quoted:

"Ok, the risk factors we have like being overweight also contribute and as well as drinking alcohol could also be a risk of cancer of the breast and the use of oral contraceptives and also the hormone replacement therapy could also lead to breast cancer."

\section{Sub-theme: Age and genetic}

Age and genetic factors was also mentioned by some participants as contributing to breast cancer.

One participant indicated:

“... I think the cause of cancer can be hereditary, meaning if 12 there are too many people in your family with breast cancer your risk is higher."

Another participant indicated:

"Age is also contributing to breast cancer because as you get older your immunity goes down and you are at higher risk for developing breast cancer."

\section{Sub-themes: Sun exposure}

The following was stated by the participant when she was asked the risk factors of breast cancer:

"Risk for me to develop breast cancer its depend because sometimes this disease is an inherited disease and normally caused by different things... 'aaa' let me say by different for example if you are exposed to the sun on top part of your breast you develop breast discharges."

Another participant stated:

"You can get breast cancer when you wear black bras in the sun."

\section{Discussion}

\subsection{Awareness of breast cancer}

The current study reveals that most participants are unemployed and did not complete secondary education. However most respondents were aware of breast examination and types of breast screening. In this study participants were able to define breast cancer and have awareness of the necessity of breast examination and screening. This implies that that although most participants are not educated their awareness of breast cancer is higher. Similarly, study conducted in Windhoek, Namibia by Mowa ${ }^{[11]}$ indicated that most $(78 \%)$ of women had awareness of breast cancer. However, current study findings are contradicts the findings from the study conducted in Zimbabwe, where $80 \%$ respondents indicated that they were not aware of breast screening for breast cancer early detection. ${ }^{[13]}$ Moreover, contrary findings were reported in the study conducted in Ghana which found out that respondent's awareness of breast cancer was mostly linked to their level of education and women with low level of educational were much more predisposed to the disease due to their lack of awareness. ${ }^{[12]}$ Another contrary findings were reported in the study conducted in Angola by Jemal et al. ${ }^{[10]}$ were female students were not aware of the fact that breast cancer is one of the most prevalent causes of death in women in Angola. The study further indicated that most of participants were not aware of signs and symptoms of breast cancer, for example change in color or shape of the nipple. ${ }^{[10]}$ Another study conducted in Iran by Harirchi et al. ${ }^{[14]}$ also reported a low level of awareness about breast 
cancer screening method among population of $32.2 \%$.

\subsection{Perception on breast cancer}

The current study findings showed that women perceive breast cancer as a very serious and dangerous disease, but it could be a curable disease when diagnosed earlier. The current study findings also showed that respondents perceived breast cancer as a killing disease if it's left untreated or diagnosed at very late stage. Around the world women perceived breast cancer differently.Study conducted in Iran indicated that more than two-thirds of women held misperception that big breasts increased the likelihood of developing breast cancer $(67 \%)$ while some believed that painful lumps are indicative of cancer (59\%). A study in Kenya identified that women perceived breast cancer to be a spiritual illness, something that lies in God's hands and God is the only person who can cure the disease. ${ }^{[15]}$ The women also believed it to be a curse that is created by the devil and can only be treated by spiritual power.

While in Nigeria women perceive breast cancer as an incurable disease, diagnose in breast cancer implies death. ${ }^{[7]}$ Begum et al.' study ${ }^{[16]}$ in Bangladesh revealed that women still have lot of misconception and disbelieve regarding breast cancer. Most of people believe that cancer is non treatable disease and there is not much they can do to prevent it and its death. While in the study conducted in Nigeria participants perceive breast cancer as an chronic, incurable disease and they believe that diagnose in breast cancer implies death. ${ }^{[17]}$ However, study in Kenya indicated that women have low perception of predisposing factors to breast cancer. Furthermore, participants, believe that breast cancer is a spiritual illness caused by an evil spirit which can only be cured by God. The women also believed it to be a curse that is created by the devil and can only be treated by spiritual power. ${ }^{[7]}$ Thus, imply that women believe in superstition which hinder the prevention and seeking behavior of breast cancer.

\subsection{Knowledge about risks factor on breast cancer}

Respondents in the current study mentioned different predisposing factors that may lead to the development of breast cancer. Thus implies that respondents showed a good knowledge. Similar findings were reported by Abdulrahman and
Rahman, ${ }^{[18]}$ most women $(94.9 \%)$ considered past history of breast cancer as a risk factor. Thus, concede with Thomson, ${ }^{[19]}$ who stated that if a women had already personal history of cancer in one breast, she has a greater chance of developing a new cancer.

Contrary findings were reported in the study conducted in UK indicated that women had limited knowledge on risk factors and breast related symptoms, only $23 \%$ correctly indicated that 1 in 10 have a chance to develop breast cancer. Less than one third recognized the role of advanced age as potential risk factors. ${ }^{[20]}$ Risk factors is defined as anything that can increase a person's chance of getting a disease such as breast cancer. ${ }^{[19]}$ Alcohol intake and smoking increase the chances of developing breast cancer. Women who started smoking before their first menstrual period were $60 \%$ at risk of being diagnosed with breast cancer than who never smoke. ${ }^{[21]}$ It was also stated that genetic or hereditary factors are some of the risk factors contributing to breast cancer. The study conducted by Anton ova, Aronson and Mueller, ${ }^{[22]}$ indicated that women who have a family history of breast cancer are at high risk for breast cancer than those without such a history. Age was also mentioned as one of the risk factor to breast cancer. ${ }^{[18]}$

\section{Conclusions}

The study findings conclude that most participants are aware of breast cancer such as definition as well as the importance of breast examination and screening. Moreover, the study revealed the mixed perception regarding breast cancer such breast cancer as a disease that can be treated or breast cancer as dangerous disease which lead to death. Contributing factors for breast cancer such as alcohol, smoking, hormonal treatment, age and hereditary factors were also unveiled during this current study. The study recommends the strengthening of breast cancer awareness program in the Ministry of Health and Social Services in order to disseminate information regarding breast cancer. The researchers recommend that further similar studies should be conducted in other regions with bigger sample.

\section{CONFLicts OF INTEREST Disclosure}

The authors declare they have no conflicts of interest.

\section{REFERENCES}

[1] Berkiten A, Sahin NH, Sahin FM, et al. Meta Analysis of Studies about Breast Self Examination between 2000-2009 in Turkey. Asian Pacific Journal of Cancer Prevention. 2012; 13(7): 3389-3397. PMid: 22994766. https ://doi .org/10.7314/APJCP. 2012.13. 7.3389

Published by Sciedu Press
[2] The American Cancer Society. Cancer facts and figures. 2017 Available from: https://www.cancer.org/content/dam/can cer-org/research/cancer-facts-and-statistics/annu al-cancer-facts-and-figures/2017/cancer-facts-and -figures-2017.pdf

[3] Han-Byoel L, Wonshik H. Unique Features of Young Age Breast Can- 
cer and Its Managementof Health. J Breast Cancer. 2014; 17(4). PMid: 25548576. https://doi.org/10.4048/jbc.2014.17.4.301

[4] Opoku SY, Benwell M, Yarney J. Knowledge, attitudes, beliefs, behaviour and breast cancer screening practices in Ghana, West Africa. PanAfr Med J. 2012; 11: 28.

[5] Rigi DN, Khojasteh F, Bandani F. Breast cancer and breast self selfexamination: acknowledgement and attitude based on health belief model in literature and engineering colleges of Sistan Balochestsn University female students. Iran J Breast Dis. 2011; 5: 65-73.

[6] Ferlay J, Soerjomataram I, Dikshit R, et al. Cancer incidence and mortality worldwide: sources, methods and major patterns in GLOBOCAN 2012. Int J Cancer. 2012; 359-86. PMid: 25220842. https://doi.org/10.1002/ijc. 29210

[7] Muthoni A, Miller AN. An exploration of rural and urban Kenyan women's knowledge and attitudes regarding breast cancer and breast cancer early detection measures. Health Care for Women International. 2013; 801-816. PMid: 20677038. https ://doi.org/10.1 080/07399331003628453

[8] Jones CEL, Maben J, Jack RH, et al. A systematic review of barriers to early presentation and diagnosis with breas cancer among black women. BMJ Open. 2014; 4: e004076. PMid: 24523424. https://doi.org/10.1136/bmjopen-2013-004076

[9] Namibia National Cancer Registry. 2016. Available from: http: //www. nampapr. com.na/?p=4574

[10] Jemal A, Bray F, Center MM, et al. Global cancer statistics. CA Cancer J Clin. 2011. PMid: 21296855. https : //doi .org/10.332 2/caac. 20107

[11] Mowa AK. An exploration of knowledge, attitudes andpractices and other breastcancer determinants influencing early detection among women in Windhoek. Doctoral dissertation. University of Namibia. 2016.

[12] Wang F, Yu ZG. Current status of breast cancer prevention in China. Chronic Dis Transl Med. 2015; 1: 2-8. PMid: 29062980. https://doi.org/10.1016/j.cdtm.2015.02.003
[13] Zaba F, Muzulu P. Cancer fight hamstrung by sosts. Zimbambwe Independent. 2013, January 27. Available from: http: //www. theindependent.co.zw/2013/11/01/cancer -fight-hamstrung-steep-costs

[14] Harirchi I, Kolahdoozan S, Karbakhsh M, et al. Twenty years of breast cancer in Iran: downstaging without a formal screening program. Ann Oncol. 2010; 22(1): 93-7. PMid: 20534622. https://doi.org/10.1093/annonc/mdq303

[15] Powe BD, Underwood S, Canales M, et al. Perceptions about breast cancer among college students: Implications for nursing education. $\mathbf{J}$ Nurs Educ. 2005 Jun; 44(6): 257-65.

[16] Begum SA, Mahmud T, Rahman T, et al. Mymensingh Med J.Knowledge, Attitude and Practice of Bangladeshi Women towards Breast Cancer: A Cross Sectional Study. Mymensingh Med J. 2019; 1: $96-104$

[17] Olayide AS, Halimat AJ, Samuel OA, et al. Level of Awareness and Knowledge of Breast Cancer in Nigeria. A Systematic Review. Ethiop J Health Sci. 2017; 27(2): 163-174. PMid: 28579712. https://doi.org/10.4314/ejhs.v27i2.9

[18] Abdulrahman GO, Rahman GA . Epidemiology of Breast Cancer in Europe and Africa. Journal of Cancer Epidemiology. 2012; 2012: 1-5. PMid: 22693503. https : //doi.org/10.1155/2012/915610

[19] Thomson CA, Rock CL, Thompson PA, et al. Vegetable intake is associated with reduced breast cancer recurrence in tamoxifen users: a secondary analysis from the Women's Healthy Eating and Living Study.Breast Cancer Res Treat. 2011; 125(2): 519-27. PMid: 20607600. https ://doi .org/10.1007/s10549-010-1014-9

[20] Grunfeld EA. Women's knowledge and beliefs regarding breast cancer. Br J Cancer. 2012; 8: 222-225.

[21] Breast cancer organization. Breast Cancer Treatment Causes Severe Side Effects in Many Women. 2013. Available from: https://www . breastcancer.org/research-news/tx-cau ses-severe-side-effects-for-many

[22] Antonova L, Aronson K, Mueller CR. Stress and breast cancer: from epidemiology to molecular biology. Breast Cancer Res. 2011; 13(2): 208. PMid: 21575279. https ://doi.org/10.1186/bcr2836 